\section{A comment on the Yaremko, Blair, and Leckart report: "The orienting reflex to changes in a conceptual
stimulus dimension"}

\author{
DAVID J. ELY \\ Porterville State Hospital, Porterville, Calif. 23257
}

\begin{abstract}
Yaremko, Blair, \& Leckart (1970) reported that a number presented out of order at the end of a list of serially presented numbers produced an orienting response (OR). ORs were found to be roughly proportional to the difference between the out-of-order number and that number to appear next in the ordered series. These results were interpreted as supporting Sokolov's (1963) expectancy model.

According to their interpretation, the presentation of the ordered list set up an expectancy that the next item to appear would be one greater than the last. The presentation of a number which was not one larger than the last elicited an orienting response.

An alternative hypothesis is proposed. Here, it is suggested that presentation of a list of numbers, either in serial order or in random sequence, will specify a range of values to which ORs become attenuated through habituation. Greatest OR attenuation is hypothesized to be somewhere in the middle of the range of values. Attenuation of the $O R$ is hypothesized to be generalized around this value.
\end{abstract}

In the experiment reported by Yaremko et al (1970), the numbers 10 through 19 were presented serially. The numbers $9,19,21$, and 31 were test stimuli. According to the stimulus

* The views expressed here are those of the author and do not necessarily represent those of the California Department of Mental Hygiene or Portervile State Hospital. generalization analogy, and making upward adjustments for recency effects, that value which would be hypothesized to have greatest OR attenuation is the number 15 . The discrepancies of the four test stimuli from this value could be rank ordered as follows: 19, 9, 21, 31 (9 and 21 are tied for rank). It would be predicted that this is the order of OR magnitudes. Mean GSRs for this order are: $7.8,13.2,19.7$, and 25.4, respectively. It seems that the results can be as adequately accounted for by assuming that the point of greatest OR attenuation is near the middle of the range of ordered stimuli as by assuming that the point of greatest $O R$ attenuation is at the number to appear next in the ordered series.

This post hoc interpretation is, of course, inconclusive. It is suggested that in subsequent experiments test stimuli be selected which will better localize the point of greatest OR attenuation. It would also seem advisable to employ routinely a control group similar to that of Zimny, Pawlick, \& Saur (1969) in which the same series of numbers are presented in a random order.

\section{REFERENCES}

SOKOLOV. E. N. Perception and the conditioned reflex. New York: Pergamon Press, 1963.

YAREMKO, R. M., BLAIR, M. W., \& LECKART, B. T. The orienting reflex to changes in a conceptual stimulus dimension. Psychonomic Science, 1970, 21,115-116.

ZIMNY, G, H., PAWLICK, G, F., \& SAUR, D. P. Effects of stimulus order and novelty on orienting responses. Psychophy siology, 1969, 6, 166-173.

\title{
Reply to Ely
}

\author{
R. M. YAREMKO \\ San Diego State College, San Diego, Calif. 92115
}

Sokolov's (1963) neuronal model of orienting reflex (OR) habituation maintains that magnitude of the OR to a novel stimulus is simply a function of the amount of discrepancy between an "expected" stimulus and the stimulus actually delivered to the organism. For example, if $S$ receives a series of $1,000-\mathrm{Hz}$ tones, OR magnitude to a test tone, different in frequency, will be commensurate with the amount of change in frequency irrespective of the direction of that change. This relationship is, of course, subject to modification by the intrusion of dynamogenic factors which contaminate manipulations of stimulus novelty. The technique of presenting a progression of numbers followed by an out-of-sequence test stimulus (TS), first reported by Unger (1964), seems at once to allow novelty manipulations, without bothersome dynamogenic effects and to permit study of the principles of habituation to more or less "conceptual" stimuli.

In the Yaremko, Blair, \& Leckart (1970) study, we predicted that an out-of-sequence TS $(9,19,21$, or 31$)$ would evoke ORs as a function of the absolute difference between the expected stimulus $(20)$ and the observed TS. Although we found OR magnitudes to be a function primarily of the amount of discrepancy between 20 and the TS, there was a tendency for negative change TSs (9 and 19) to evoke smaller ORs. The fact that 19 evoked the smallest ORs was not surprising, since this stimulus was presented on the preceding trial, and although unexpected, was still physically familiar. As Ely suggests, however, the smaller ORs to 9 could be explained by the fact that this stimulus lies in closer proximity to the stimulus series that $S$ was already habituated to, and thus, should have a ttached to it more generalized inhibition than 31 .

While it may be a step away from parsimony to postulate generalization gradients in this kind of conceptual dimension, the possibility is worth further study. Some data 1 recently collected in our laboratory bear directly on the issue. We replicated the earlier procedure using the series 10-22 in steps of two numbers. Where 24 was expected, $S$ received the TS $9,21,27$, or 39 ( \pm 3 or \pm 15 units' change). This procedure allowed us to interpolate a TS (21) without using a physically familiar number. According to Ely's position, there should be less responding to 21 than to 27 , since generalized inhibition should be near maximum in this vicinity of the dimension. Even when ORs were compared with a base level of responding obtained by averaging responses to 18,20 , and 22 , no differences were uncovered within the small- and large-change conditions. As in the earlier study, OR magnitude to the TSs was a function of amount of change only. On balance, these findings seem best explained by the expectancy-disparity approach or a generalization of inhibition approach with the "expected" stimulus as the reference.

REFERENCES
SOKOLOV, $\mathrm{Y}$. N. Perception and the 
conditioned reflex. New York: Pergamon Press, 1963.

UNGER, S. M. Habituation of the vasoconstrictive orienting reaction. Journal of Experimental Psychology, 1964, 67, 11-18.

YAREMKO, R. M. BLAIR, M. W., \& LECKART, B. T. The orienting reflex to changes in a conceptual stimulus
dimension. Psychonomic Science, 1970, 21, 115-116.

\section{NOTE}

1. Yaremko, R. M., Keleman, K., \& Leckart, B. T. Orienting reflexes and conceptual stimuli. Unpublished investigation.

\section{Reward power and bilateral communication in conflict resolution}

\author{
SVENN LINDSKOLD \\ Ohio University, Athens, Ohio 45701 \\ and
}

JAMES T. TEDESCHI, THOMAS V. BONOMA, and BARRY R. SCHLENKER State University of New York at Albany, Albany, N.Y. 12203

Eighty female Ss served as targets of contingent promise messages sent intermittently during the course of a prisoner's dilemma game. Ss were either provided with or lacked intentional reply messages to respond to the source and faced an influencer who rewarded target compliance to her promises $0 \%, 10 \%$, $90 \%$, or $100 \%$ of the time. Ss with reply capability were more cooperative on nonpromise occasions than were no-reply Ss, but neither reply availability nor probability of reward mediation affected behavioral compliance. Postgame ratings indicated that $S s$ in the high probability of reward conditions evaluated the source as more attractive, but less potent, than the source who seldom or never kept her promises.

Recent studies have reliably found that S's compliance to contingent threats is a direct function of both punishment magnitude and the cumulated probability of punishment over an interaction sequence (cf. Tedeschi, 1970). Similar studies of promises have yielded contradictory results. Two investigations found no relationship between the probability of rewards and compliance to the promisor's requests (Lindskold, Bonoma, Schlenker, \& Tedeschi, 1971; Lindskold \& Tedeschi, 1971). However, Horai, Haber, Tedeschi, \& Smith (1970) obtained a direct relationship between probability of reward and S's compliance. Retrospective comparisons of the three promise studies indicated two possible differences in methodology which might account for the divergent findings. First, the studies which failed to establish a relationship between probability and compliance varied the probabilities between $10 \%$ and $90 \%$, while the study which found the direct relationship used probability levels of $0 \%$ and $100 \%$. It is possible that a single lie causes the target to have suspicions about all succeeding promises. Alternatively, a single reward may be sufficient to obtain relatively high levels of compliance. Secondly, in the first two studies, $S$ was required to reply to the promises with a statement of behavioral intentions, although he could choose to be truthful, untruthful, or evasive in his replies. The study which found the probability-compliance relationship did not allow $S$ to announce his behavioral intentions. Since investigations such as that of Tedeschi, Bonoma, \& Lindskold (1970) have shown the importance of such replies in achieving conflict amelioration and resolution, it is plausible that the inclusion or omission of statements of verbal commitments could change the character of the interaction and account for the contradictory findings at issue.

The above differences between studies were investigated in a 2 by 4 factorial design. Ss were targets of promises from a simulated opponent during a mixed-motive conflict. The simulated promisor offered the $S$ a reward if the latter would make the cooperative response in a two-choice situation. Ss were either given the opportunity to announce their own intentions in reply to the promisor's messages or else they could not communicate at all. The simulated promisor gave the promised reward $0 \%, 10 \%, 90 \%$, or $100 \%$ of the times the Ss made the compliant response. METHOD

The Ss were 80 female undergraduates who satisfied a requirement at the United States International University by their participation. Ss were recruited in pairs to foster the impression that each was participating with a peer. This impression was maintained throughout the interaction, although $\mathrm{E}$ simulated the play of the "other" for all Ss. Ss faced a prisoner's dilemma (PD) game panel, which included two buttons, one for the cooperative (Choice 1) and one for the competitive (Choice 2) strategy selections. A 2 by 2 payoff matrix was mounted at the top of the panel, each cell of which could be separately illuminated to provide feedback about own and "other's" payoffs following each trial. The payoffs displayed in the matrix were such that both $\mathrm{S}$ and $\mathrm{E}$ won 4 points if both cooperated, and both lost 4 points if both competed; if one cooperated while the other competed, the former lost 5 points and the latter won 5 points. Two add-subtract cumulative counters, mounted on the upper right-hand side of the panel, provided feedback regarding each player's scores. Two rows of message slots were placed on the face of the panel below the matrix. Next to each message slot was a button on the outside part of the panel (to transmit) and a light on the inside part of the panel (to receive). Signal lights served to order events and regulate play throughout the interaction.

A promise message was posted on the incoming (left) side of the S's panel and read: "If you make Choice 1 on the next trial, I will add 10 points to your counter." Ss in the bilateral communication conditions had three reply messages posted on the outgoing (right) side of their panel which read: (M1) "I will make Choice 1 on the next trial," (M2) "I will make Choice 2 on the next trial," and (M3) "I do not wish to disclose my intentions." Ss in the unilateral communication condition did not, of course, have any reply messages posted on the panel.

In order to equate the number of rewards received by Ss within cells and to establish fixed probabilities of rewards, a criterion of 10 successful promises (i.e., promises to which $\mathrm{S}$ complied by making Choice 1 ) was set. After each $S$ reached criterion, no further promises were sent to her. To establish the criterion, the $E$ predesignated that there would be two options to send the promise during each block of 10 PD trials. One successful promise per block was the criterion pace, but if the $S$ fell behind the pace, both messages per block were sent until either the pace was reestablished or the criterion of 10 successful promises was met. After each $S$ reached the criterion, no further messages were sent to her. E always made the cooperative game choice on the trial immediately following the transmission of a promise and maintained a fixed, but 